\title{
Uninterrupted Dabigatran versus Warfarin for Ablation in Atrial Fibrillation
}

\author{
Hugh Calkins, M.D., Stephan Willems, M.D., Edward P. Gerstenfeld, M.D., \\ Atul Verma, M.D., Richard Schilling, M.D., Stefan H. Hohnloser, M.D., \\ Ken Okumura, M.D., Ph.D., Harvey Serota, M.D., Matias Nordaby, M.D., \\ Kelly Guiver, M.Sc., Branislav Biss, M.D., Marc A. Brouwer, M.D., Ph.D., \\ and Massimo Grimaldi, M.D., Ph.D., for the RE-CIRCUIT Investigators*
}

A BSTRACT

\section{BACKGROUND}

Catheter ablation of atrial fibrillation is typically performed with uninterrupted anticoagulation with warfarin or interrupted non-vitamin $\mathrm{K}$ antagonist oral anticoagulant therapy. Uninterrupted anticoagulation with a non-vitamin $\mathrm{K}$ antagonist oral anticoagulant, such as dabigatran, may be safer; however, controlled data are lacking. We investigated the safety of uninterrupted dabigatran versus warfarin in patients undergoing ablation of atrial fibrillation.

\section{METHODS}

In this randomized, open-label, multicenter, controlled trial with blinded adjudicated end-point assessments, we randomly assigned patients scheduled for catheter ablation of paroxysmal or persistent atrial fibrillation to receive either dabigatran (150 mg twice daily) or warfarin (target international normalized ratio, 2.0 to 3.0). Ablation was performed after 4 to 8 weeks of uninterrupted anticoagulation, which was continued during and for 8 weeks after ablation. The primary end point was the incidence of major bleeding events during and up to 8 weeks after ablation; secondary end points included thromboembolic and other bleeding events.

\section{RESULTS}

The trial enrolled 704 patients across 104 sites; 635 patients underwent ablation. Baseline characteristics were balanced between treatment groups. The incidence of major bleeding events during and up to 8 weeks after ablation was lower with dabigatran than with warfarin (5 patients [1.6\%] vs. 22 patients [6.9\%]; absolute risk difference, -5.3 percentage points; $95 \%$ confidence interval, -8.4 to -2.2 ; $\mathrm{P}<0.001)$. Dabigatran was associated with fewer periprocedural pericardial tamponades and groin hematomas than warfarin. The two treatment groups had a similar incidence of minor bleeding events. One thromboembolic event occurred in the warfarin group.

\section{CONCLUSIONS}

In patients undergoing ablation for atrial fibrillation, anticoagulation with uninterrupted dabigatran was associated with fewer bleeding complications than uninterrupted warfarin. (Funded by Boehringer Ingelheim; RE-CIRCUIT ClinicalTrials.gov number, NCT02348723.)
From Johns Hopkins Medical Institutions, Baltimore (H.C.); Department of CardiologyElectrophysiology, University Heart Center Hamburg, Hamburg (S.W.), Department of Cardiology, J.W. Goethe University, Frankfurt (S.H.H.), and Boehringer Ingelheim Pharma, Ingelheim am Rhein (M.N.) - all in Germany; Section of Cardiac Electrophysiology, University of California, San Francisco, San Francisco (E.P.G.); University of Toronto, Toronto (A.V.); Barts Heart Centre, Saint Bartholomew's Hospital, London (R.S.), and Biometrics and Data Sciences Department, Boehringer Ingelheim, Bracknell (K.G.) both in the United Kingdom; Division of Cardiology, Saiseikai Kumamoto Hospital Cardiovascular Center, Kumamoto, Japan (K.O.); St. Louis Heart and Vascular, St. Louis (H.S.); Department of Clinical Operations, Boehringer Ingelheim Regional Center Vienna, Vienna (B.B.); Department of Cardiology, Radboud University Medical Center, Nijmegen, the Netherlands (M.A.B.); and Cardiology Department, Miulli Hospital, Acquaviva delle Fonti, Italy (M.G.). Address reprint requests to Dr. Calkins at Johns Hopkins Medical Institutions, Sheikh Zayed Tower 7125R, 1800 Orleans St., Baltimore, MD, or at hcalkins@jhmi.edu.

*A complete list of investigators in the RE-CIRCUIT (Randomized Evaluation of Dabigatran Etexilate Compared to Warfarin in Pulmonary Vein Ablation: Assessment of an Uninterrupted Periprocedural Anticoagulation Strategy) trial is provided in the Supplementary Appendix, available at NEJM.org.

This article was published on March 19, 2017, at NEJM.org.

N Engl J Med 2017;376:1627-36. DOI: $10.1056 /$ NEJMoal701005

Copyright $@ 2017$ Massachusetts Medical Society. 


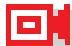

A Quick Take is available at NEJM.org atheter ablation of atrial Fibrillation is a well-established treatment for symptomatic atrial fibrillation. Guidelines have incorporated catheter ablation of symptomatic atrial fibrillation as a class 1 or 2 indication, depending on previous antiarrhythmic treatment and type of atrial fibrillation. ${ }^{1-3}$ The most important complications associated with ablation of atrial fibrillation are periprocedural stroke or transient ischemic attack (TIA) and cardiac tamponade. $^{3}$

Systemic anticoagulation before, during, and after ablation is important in reducing the risk of periprocedural cerebrovascular events. ${ }^{3}$ To minimize these risks, heparin should be administered during ablation to maintain an activated clotting time of more than 300 seconds. However, there is less consensus on preprocedural and postprocedural anticoagulation management. ${ }^{3}$ Uninterrupted vitamin $\mathrm{K}$ antagonism during the time of ablation of atrial fibrillation is associated with a lower risk of periprocedural bleeding and stroke than stopping vitamin $\mathrm{K}$ antagonism and bridging with low-molecular-weight heparin.,

Preliminary safety data are available from observational studies of cohorts of patients undergoing catheter ablation with uninterrupted nonvitamin $\mathrm{K}$ antagonist oral anticoagulant therapy ${ }^{6-17}$; these data are supported by recent meta-analyses comparing periprocedural non-vitamin $\mathrm{K}$ antagonist oral anticoagulant therapy with uninterrupted vitamin $\mathrm{K}$ antagonists. ${ }^{18,19}$ The limited systematic, prospective clinical data for non-vitamin $\mathrm{K}$ antagonist oral anticoagulants in the context of catheter ablation of atrial fibrillation poses a management challenge. ${ }^{3,4,6-20}$ One exploratory controlled trial involving 248 patients showed that the uninterrupted use of a factor $\mathrm{Xa}$ inhibitor was feasible; the incidences of thromboembolic events and major bleeding events were low and were similar to those with uninterrupted doseadjusted vitamin $\mathrm{K}$ antagonist therapy. ${ }^{21}$

Dabigatran etexilate (dabigatran) has efficacy and safety outcomes similar or superior to those of warfarin for stroke prevention in patients with atrial fibrillation. ${ }^{22,23}$ The favorable safety and efficacy profile of dabigatran has been consistently confirmed by practice-based evidence from multiple sources, including two Food and Drug Administration studies and several independent scientific groups. ${ }^{24-27}$ The RE-CIRCUIT (Randomized Evaluation of Dabigatran Etexilate Compared to
Warfarin in Pulmonary Vein Ablation: Assessment of an Uninterrupted Periprocedural Anticoagulation Strategy) trial was a prospective, randomized trial investigating the safety and efficacy of a periprocedural anticoagulation regimen with uninterrupted dabigatran as compared with uninterrupted warfarin in patients undergoing ablation of atrial fibrillation.

\section{METHODS}

\section{TRIAL DESIGN}

The RE-CIRCUIT trial was a randomized, openlabel, multicenter, controlled trial with blinded adjudicated end-point assessments involving patients with nonvalvular atrial fibrillation undergoing ablation of atrial fibrillation. The trial was conducted in accordance with the provisions of the Declaration of Helsinki and the International Conference on Harmonisation Good Clinical Practice guidelines. ${ }^{28,29}$ The protocol (available with the full text of this article at NEJM.org) was approved by the institutional review board or independent ethics committee at each participating center. Written informed consent was obtained from all the patients before trial entry. The trial was conducted under the guidance of a steering committee, and data (both adjudicated and nonadjudicated) were scrutinized by an independent data and safety monitoring committee. All primary and secondary end points were adjudicated by an independent committee whose members were unaware of the trial-group assignments. The trial was designed by the authors in collaboration with the sponsor (Boehringer Ingelheim). The authors vouch for the accuracy and completeness of the data and analyses and for the fidelity of the trial to the protocol. The development of the manuscript was led by the first author, in collaboration with all the authors. All the authors made the decision to submit the manuscript for publication. Assistance with manuscript preparation was provided by a medical writer funded by the sponsor.

\section{TRIAL POPULATION}

Full inclusion and exclusion criteria are listed in the protocol. Patients 18 years of age or older were included in the trial if they had paroxysmal or persistent nonvalvular atrial fibrillation with planned ablation of atrial fibrillation, had documented atrial fibrillation within 24 months be- 
fore screening, and were eligible for treatment with dabigatran (150 mg twice daily) according to the local prescribing information. Key exclusion criteria were permanent atrial fibrillation, atrial fibrillation secondary to an obvious reversible cause, and valvular atrial fibrillation.

\section{TREATMENT}

Patients were randomly assigned in blocks (1:1 ratio) to receive either dabigatran $(150 \mathrm{mg}$ twice daily) or warfarin (combination of 1,3 , and $5 \mathrm{mg}$, with dose adjustment for a target international normalized ratio [INR] of 2.0 to 3.0), with no stratification. The Rosendaal method was used to evaluate the percentage of time that a patient's INR was in the therapeutic range. ${ }^{30}$ Patients who had previously taken a vitamin $\mathrm{K}$ antagonist and were assigned to the warfarin group started the trial medication when the INR was less than 3.0 ( $<2.6$ in patients at Japanese sites $\geq 70$ years of age); dabigatran treatment was initiated when a patient's INR was less than 2.0.

The trial consisted of four sequential periods: a screening period of 0 to 2 weeks; a preablation treatment period of 4 to 8 weeks, to achieve the desired stable anticoagulation range in patients receiving warfarin; a postablation treatment period of 8 weeks (starting with the ablation procedure); and a follow-up period of 1 week (Fig. S1 in the Supplementary Appendix, available at NEJM .org). All the patients were to undergo preablation transesophageal echocardiography to rule out left atrial thrombi. The morning dose of dabigatran was taken on the day of the ablation at the patient's usual scheduled time. Ablation was performed with uninterrupted anticoagulation treatment, and anticoagulation was continued for 8 weeks after the procedure. Unfractionated heparin was administered after the placement of femoral sheaths before or immediately after transseptal puncture. During the ablation procedure, achieving and maintaining an activated clotting time of more than 300 seconds was recommended. Dabigatran administration was continued in the evening of the procedure at the scheduled time, with a minimum delay of 3 hours after sheath removal and achievement of hemostasis.

The procedure for ablation of atrial fibrillation was performed according to the recommendations and guidelines of a 2012 expert consensus statement. ${ }^{3}$ Patients underwent ablation at the discretion of the attending electrophysiologist; the proce- dure included pulmonary-vein isolation and any additional substrate-modification approach typically performed in that laboratory. Radiofrequency energy was typically used; however, other energy sources such as cryoablation or laser ablation were allowed.

All the patients were to have a follow-up visit 1 week after the treatment with trial medication ended, which was 8 weeks after the ablation procedure. Any decision to continue anticoagulation with nontrial medication after the follow-up period was based on the recommendations and guidelines ${ }^{1,3}$ and at the treating physician's discretion.

\section{TRIAL END POINTS \\ Primary End Point}

The primary end point was the incidence of adjudicated major bleeding events as defined by the International Society on Thrombosis and Hemostasis (ISTH). ${ }^{31}$ Major bleeding events were considered from the start of the ablation procedure, from the first femoral puncture to up to 8 weeks after ablation. Prespecified subgroups were analyzed with the use of descriptive statistics (exploratory testing). Information on major bleeding events requiring medical attention (e.g., compression of a puncture site, the use of vitamin $\mathrm{K}$ or prothrombin complex concentrates, or a procedure or surgical intervention) was also collected.

\section{Secondary End Points}

The secondary efficacy and safety end points were the incidence of the following events during and up to 8 weeks after ablation: a composite of stroke, systemic embolism, or TIA; minor bleeding events; and a composite of major bleeding events and thromboembolic events (stroke, systemic embolism, or TIA). Minor bleeding events were defined as clinical bleeding events that did not fulfill ISTH criteria for major bleeding events.

\section{ADVERSE EVENTS}

All adverse events throughout the trial period were recorded and analyzed. An adverse event was defined as any untoward medical occurrence in a patient administered a trial treatment. A severe adverse event was defined as an event that was incapacitating or caused an inability to work or perform usual activities. Data on adverse events were collected from the time of signing of the informed consent, and events were further classified 
as serious or nonserious during the course of the trial. Efficacy and safety end points were reported only as adverse events. Because efficacy and safety end points could also be serious adverse events, these events were not reported as serious adverse events on the serious-adverse-event form to the sponsor and consequently were not reported in an expedited manner to the competent authorities.

\section{STATISTICAL ANALYSIS}

The trial had a planned enrollment of at least 290 patients per treatment group undergoing ablation across 114 international sites. Calculations were performed with the use of nQuery Advisor, version 7.0 (Statistical Solutions). The trial was exploratory because the sample required to provide sufficient power to establish formal noninferiority with an acceptable upper limit of the $95 \%$ confidence interval (e.g., 1.5) would have made the trial unfeasible ( $>2000$ patients per group). On the basis of multiple scenarios, it was decided that a minimum of 290 patients who could be evaluated per treatment group would be enough to provide clinically meaningful information. Under the assumption of no difference in absolute rates of major bleeding events between the two treatment groups, the chosen total sample of 580 would produce a confidence interval for the difference between dabigatran and warfarin with an upper limit of $2.48 \%$, if the absolute rate of major bleeding events were $0.59 \%$. If the absolute rate of major bleeding events were $4.55 \%$, the upper limit of the confidence interval of the difference would have been $4.98 \% .^{32}$ To minimize any potential bias, a central randomization strategy using computer-generated sequences was implemented through an interactive computerized response system.

To provide a statistical framework to identify differences between the treatment groups, nominal $\mathrm{P}$ values were determined. The primary and secondary end point analyses were based on the ablation set. The ablation set included all randomly assigned patients who had taken at least one dose of trial drug and who had undergone the ablation procedure. Point estimates for the incidence of major bleeding events and their twosided $95 \%$ confidence intervals, based on the normal approximation of independent binomial distribution without stratification, were determined. The risk difference between dabigatran and warfarin was calculated, and the two-sided $95 \%$ con- fidence interval and corresponding $\mathrm{P}$ value were estimated. In general, adverse events were analyzed descriptively. The treated set (all randomly assigned patients who had taken $\geq 1$ dose of trial drug) were included in the safety analysis, and the summaries were based on the actual treatment received. Statistical analysis and reporting of adverse events concentrated on events that emerged during treatment.

\section{RESULTS}

\section{TRIAL PARTICIPANTS}

The trial enrolled 704 patients across 104 sites in 11 countries from April 2015 through July 2016, of whom 678 patients underwent randomization. The ablation set included 635 patients (317 in the dabigatran group and 318 in the warfarin group) (Fig. 1). Demographic and clinical characteristics were well balanced between the treatment groups at baseline (Table 1). Dabigatran adherence was calculated on the basis of the number of capsules taken; nonadherence was recorded when adherence was not between $80 \%$ and $120 \%$ of the expected dose. Warfarin adherence was monitored with the use of the INR. The mean adherence with dabigatran was $97.6 \%$, and patients receiving INR-adjusted warfarin were within the guideline-defined target INR range (2.0 to 3.0) for $66 \%$ of the time overall. The majority of patients $(86.1 \%$ in the dabigatran group and $84.3 \%$ in the warfarin group) received trial medication for at least 8 weeks after ablation, and more than $98 \%$ received trial medication for at least 6 weeks, with no meaningful difference between the treatment groups (Table S1 in the Supplementary Appendix).

\section{PRIMARY END POINT}

There were 27 patients overall with adjudicated major bleeding events during and up to 8 weeks after ablation. The percentage of patients with major bleeding events was significantly lower in the dabigatran group than in the warfarin group (5 patients [1.6\%] vs. 22 patients [6.9\%]; absolute risk difference, -5.3 percentage points; $95 \%$ confidence interval $[\mathrm{CI}],-8.4$ to $-2.2 ; \mathrm{P}<0.001$ ). The relative risk reduction versus warfarin was $77.2 \%$. The robustness of the primary end point was supported by a Cox proportional-hazards analysis showing a hazard ratio of 0.22 for dabigatran versus warfarin (95\% CI, 0.08 to 0.59 ) 


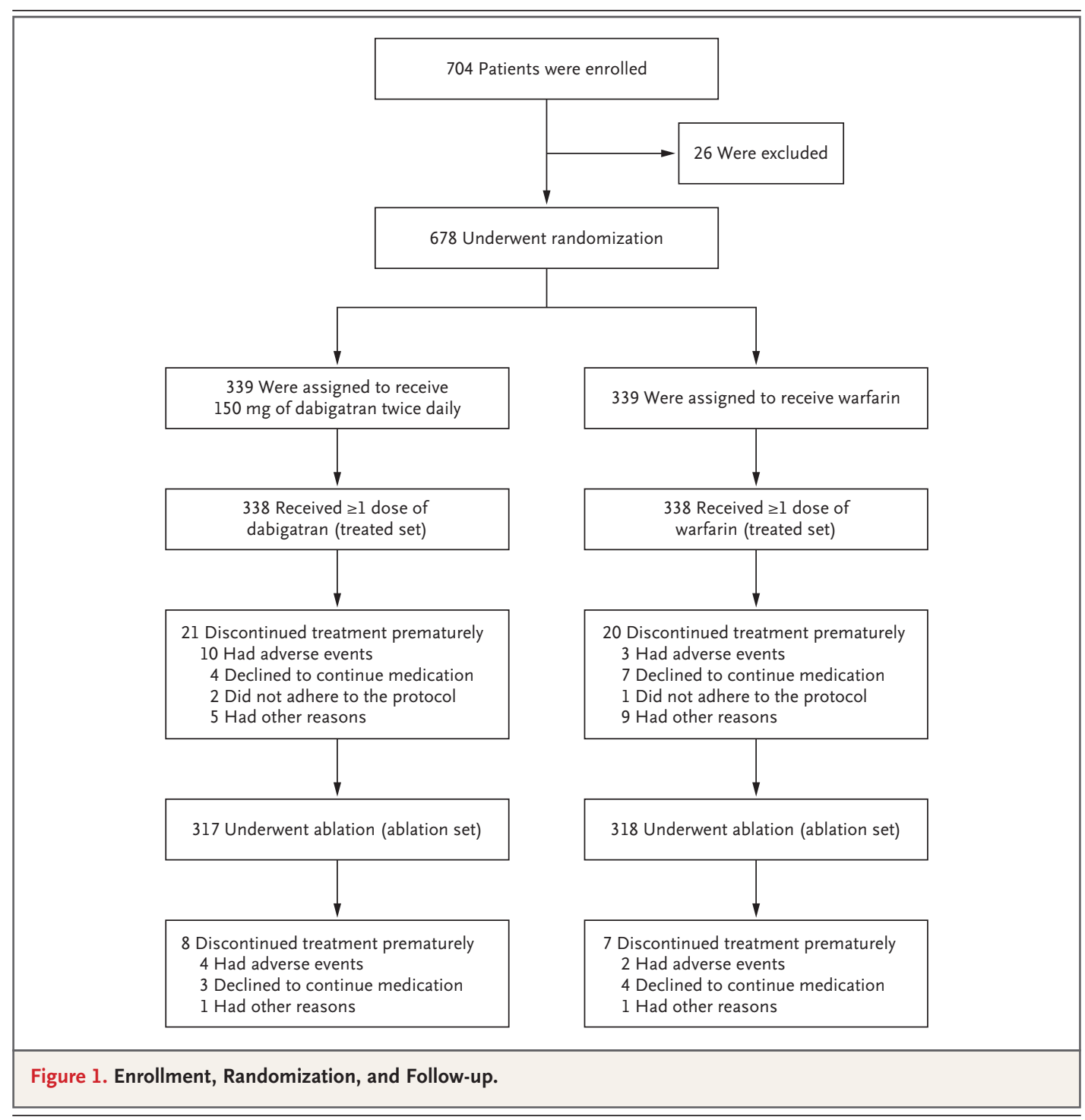

(Fig. 2). The numbers of major bleeding events in the two groups were as follows: pericardial tamponade, 1 event in the dabigatran group and 6 events in the warfarin group; pericardial effusion, 1 and 0 ; groin bleeding, 2 and 2; groin hematoma, 0 and 8; intracranial bleeding, 0 and 2; pseudoaneurysm, 0 and 1; gastrointestinal bleeding, 1 and 2; and hematoma, 0 and 2 (Table S2 in the Supplementary Appendix). In particular, dabigatran was associated with fewer major bleeding events than warfarin from the time of ablation to 7 days after ablation (4 vs. 17 major bleeding events) (Table S3 in the Supplementary Appendix).

In the dabigatran group, 131 patients (41.3\%) received the final dose of dabigatran less than 4 hours before the transseptal puncture, 116 pa- tients (36.6\%) 4 to less than 8 hours before the puncture, and 62 patients (19.6\%) 8 hours or more before the puncture. The information was lacking for 8 patients. Two major bleeding events occurred in the "within 4 hours" group and 3 major bleeding events in the "within 4 to 8 hours" group. In the warfarin group, the mean INR at the time of the ablation was similar in patients with major bleeding events and those without such events (2.4 and 2.3, respectively). The mean intraprocedural activated clotting time in patients with major bleeding events was 374 seconds in the dabigatran group and 314 seconds in the warfarin group; in patients without major bleeding events, the time was 329 seconds in the dabigatran group and 344 seconds in the warfarin group. 


\begin{tabular}{|c|c|c|}
\hline Characteristic & $\begin{array}{l}\text { Dabigatran, } \\
150 \text { mg Twice Daily } \\
\text { ( } \mathrm{N}=317)\end{array}$ & $\begin{array}{l}\text { Warfarin } \\
(N=318)\end{array}$ \\
\hline Age $-y r$ & $59.1 \pm 10.4$ & $59.3 \pm 10.3$ \\
\hline Male sex — no. (\%) & $230(72.6)$ & $245(77.0)$ \\
\hline Mean body-mass index' & 28.5 & 28.8 \\
\hline Mean $\mathrm{CHA}_{2} \mathrm{DS}_{2}$-VASc score & 2.0 & 2.2 \\
\hline \multicolumn{3}{|l|}{ Activated clotting time } \\
\hline No. of patients analyzed & 312 & 308 \\
\hline Mean - sec & 330 & 342 \\
\hline \multicolumn{3}{|l|}{ Medical history — no. (\%) } \\
\hline Congestive heart failure & $31(9.8)$ & $34(10.7)$ \\
\hline Left ventricular dysfunction & $25(7.9)$ & $23(7.2)$ \\
\hline Coronary artery disease & $32(10.1)$ & $48(15.1)$ \\
\hline Percutaneous coronary intervention & $16(5.0)$ & $19(6.0)$ \\
\hline Previous myocardial infarction & $10(3.2)$ & $15(4.7)$ \\
\hline Hypertension & $166(52.4)$ & $177(55.7)$ \\
\hline Previous stroke & $10(3.2)$ & $9(2.8)$ \\
\hline Previous major bleeding or predisposition & $3(0.9)$ & $4(1.3)$ \\
\hline Previous GI bleeding or gastritis & $24(7.6)$ & $21(6.6)$ \\
\hline Renal disease & $7(2.2)$ & $14(4.4)$ \\
\hline Diabetes mellitus & $30(9.5)$ & $34(10.7)$ \\
\hline \multicolumn{3}{|l|}{ Atrial fibrillation - no. (\%) } \\
\hline Paroxysmal & $213(67.2)$ & 219 (68.9) \\
\hline Persistent & $86(27.1)$ & $81(25.5)$ \\
\hline Long-standing persistent & $18(5.7)$ & $18(5.7)$ \\
\hline \multicolumn{3}{|l|}{ Medication use - no. (\%)』 } \\
\hline Vitamin $\mathrm{K}$ antagonists & $95(28.1)$ & $86(25.4)$ \\
\hline Dabigatran & $45(13.3)$ & $36(10.7)$ \\
\hline Rivaroxaban & $29(8.6)$ & $29(8.6)$ \\
\hline Apixaban & $21(6.2)$ & $30(8.9)$ \\
\hline Edoxaban & $3(0.9)$ & 0 \\
\hline NSAIDs & $66(19.5)$ & $78(23.1)$ \\
\hline Proton-pump inhibitors & $73(21.6)$ & $79(23.4)$ \\
\hline Statins & $106(31.4)$ & $101(29.9)$ \\
\hline Beta-blockers & $195(57.7)$ & $204(60.4)$ \\
\hline
\end{tabular}

* Plus-minus values are means \pm SD. The ablation set included all randomly assigned patients who had taken at least one dose of trial drug and who had undergone the ablation procedure. GI denotes gastrointestinal, and NSAID nonsteroidal antiinflammatory drug.

$\dagger$ The body-mass index is the weight in kilograms divided by the square of the height in meters.

7 The $\mathrm{CHA}_{2} \mathrm{DS}_{2}$-VASc score reflects the risk of stroke among patients with atrial fibrillation. Scores range from 0 to 9 , with higher scores indicating greater risk.

$\int$ Values are for the treated set (338 patients in each group).

The New England Journal of Medicine 
Subgroup analyses of the incidence of adjudicated major bleeding events during and up to 8 weeks after ablation were generally consistent with the overall results favoring the dabigatran group. Major bleeding events were, in general, less frequent in the dabigatran group irrespective of sex, age, creatinine clearance, $\mathrm{CHA}_{2} \mathrm{DS}_{2}$-VASc score (which reflects the risk of stroke among patients with atrial fibrillation), status with respect to previous hypertension, type of ablation, geographic region, and body-mass index (Fig. S2 in the Supplementary Appendix).

In the dabigatran group, 4 patients with a major bleeding event required medical action; only the pericardial tamponade required procedural intervention (namely, pericardial drainage). Idarucizumab, a specific dabigatran-reversal agent, was not used to treat any of the patients in the dabigatran group who required medical attention. In the warfarin group, 21 patients with a major bleeding event required medical attention, and 11 of these patients required an intervention (6 underwent pericardial drainage, 3 underwent surgical repair of the femoral artery, 1 underwent polyp removal, and 1 received a retroperitoneal intervention) (Table S3 in the Supplementary Appendix).

\section{SECONDARY END POINTS}

There were no events of stroke, systemic embolism, or TIA in the dabigatran group and only one event (TIA) in the warfarin group from the time of ablation until 8 weeks after ablation. The incidence of minor bleeding events was similar in the two treatment groups (59 patients [18.6\%] in the dabigatran group and 54 patients $[17.0 \%]$ in the warfarin group). The composite incidence of major bleeding events and thromboembolic events (stroke, systemic embolism, or TIA) was lower in the dabigatran group than in the warfarin group (5 patients [1.6\%] vs. 23 patients [7.2\%]).

\section{ADVERSE EVENTS}

Serious adverse events were reported in $18.6 \%$ of the patients in the dabigatran group and $22.2 \%$ of the patients in the warfarin group (Table S4 in the Supplementary Appendix). No fatal events were reported in either treatment group. In addition, dabigatran was associated with fewer severe adverse events (3.3\% with dabigatran and $6.2 \%$ with warfarin). The incidence of adverse events leading to discontinuation of trial treatment was

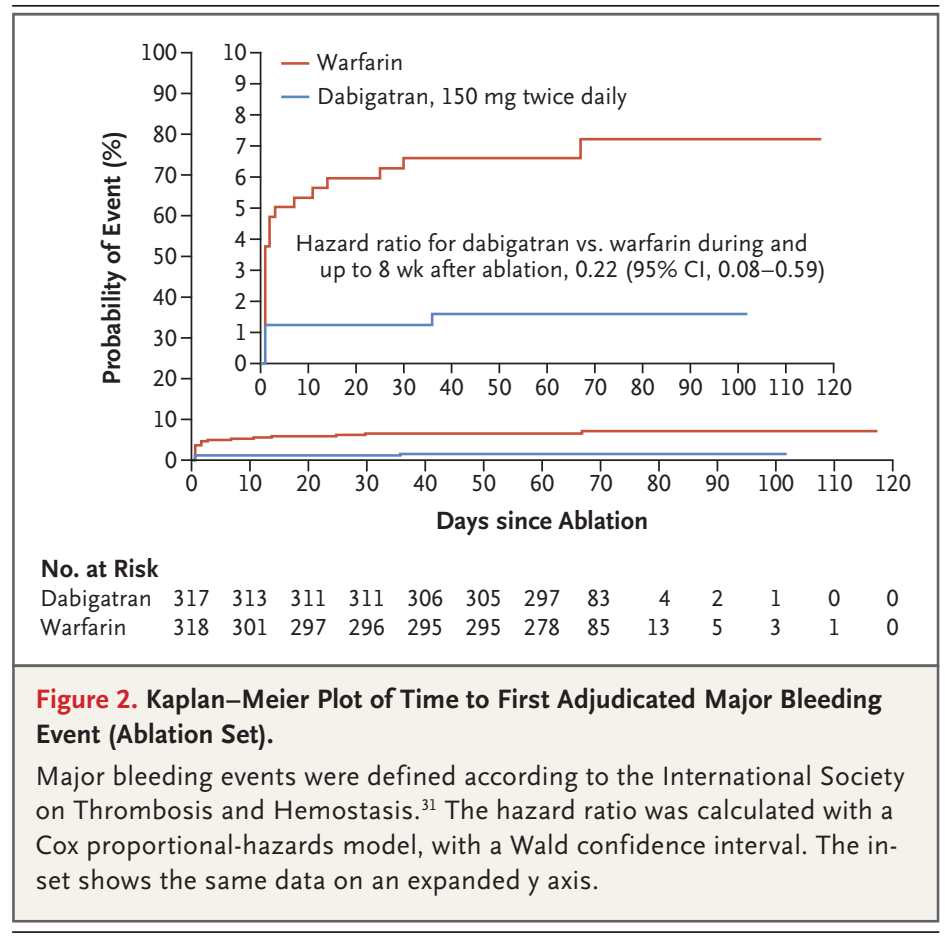

low in both groups $(5.6 \%$ with dabigatran and $2.4 \%$ with warfarin) (Table 2). In 15 patients, the ablation procedure was canceled or postponed for a variety of investigator-identified reasons; a thrombus identified on transesophageal echocardiography was reported in 1 patient (Table S5 in the Supplementary Appendix).

\section{DISCUSSION}

This multicenter, randomized, controlled trial assessed the safety and efficacy of uninterrupted dabigatran as compared with warfarin as a periprocedural anticoagulant in patients undergoing ablation of atrial fibrillation. Dabigatran was associated with a significantly lower rate of major bleeding events than INR-adjusted warfarin, and there were no differences in the incidence of stroke or systemic embolism.

One of the most feared complications of ablation of atrial fibrillation is stroke. Previous clinical trials have shown that continuous vitamin $\mathrm{K}$ antagonism is associated with fewer embolic events than interrupted treatment. ${ }^{3,4}$ However, data on the use of non-vitamin $\mathrm{K}$ antagonist oral anticoagulants around the time of catheter ablation are limited. ${ }^{15-17,21}$ Most electrophysiologists have interrupted the dose of non-vitamin $\mathrm{K}$ an- 


\begin{tabular}{|c|c|c|c|}
\hline Event & $\begin{array}{l}\text { Dabigatran, } \\
150 \text { mg Twice Daily } \\
\text { (N=338) }\end{array}$ & $\begin{array}{l}\text { Warfarin } \\
(\mathrm{N}=338) \\
\text { nber (percen }\end{array}$ & $\begin{array}{c}\text { Total } \\
(N=676)\end{array}$ \\
\hline Any adverse event & $225(66.6)$ & $242(71.6)$ & $467(69.1)$ \\
\hline Severe adverse event $\uparrow$ & $11(3.3)$ & $21(6.2)$ & $32(4.7)$ \\
\hline Adverse event leading to treatment discontinuation & $19(5.6)$ & $8(2.4)$ & $27(4.0)$ \\
\hline Serious adverse event & $63(18.6)$ & $75(22.2)$ & $138(20.4)$ \\
\hline Fatal adverse event & 0 & 0 & 0 \\
\hline Immediately life-threatening event & $1(0.3)$ & $2(0.6)$ & $3(0.4)$ \\
\hline $\begin{array}{l}\text { Event that resulted in clinically significant or } \\
\text { persistent disability or incapacity }\end{array}$ & 0 & $1(0.3)$ & $1(0.1)$ \\
\hline Event that required hospitalization & $26(7.7)$ & $34(10.1)$ & $60(8.9)$ \\
\hline Event that prolonged hospitalization & $13(3.8)$ & $22(6.5)$ & $35(5.2)$ \\
\hline Other: & $29(8.6)$ & $27(8.0)$ & $56(8.3)$ \\
\hline \multicolumn{4}{|c|}{$\begin{array}{l}\text { The treated set included all randomly assigned patients who had taken at least one dose of trial drug. A patient may be counted as having } \\
\text { an event that fulfills more than one seriousness criterion. Percentages were calculated with the total number of patients per treatment as } \\
\text { the denominator. } \\
\text { A severe adverse event was defined as an event that is incapacitating or causes an inability to work or perform usual activities. } \\
\text { The "other" category included events deemed to be serious by the investigator in that they were important medical events that, after appro- } \\
\text { priate medical judgment, may have required medical or surgical intervention to prevent any of the outcomes mentioned previously. }\end{array}$} \\
\hline
\end{tabular}

tagonist oral anticoagulants before catheter ablation, out of concern that bleeding complications could lead to worse outcomes in the presence of an "irreversible" anticoagulant. High-risk patients receiving non-vitamin $\mathrm{K}$ antagonist oral anticoagulants often had their treatment changed over to vitamin $\mathrm{K}$ antagonists periprocedurally, so that ablation could be performed with continuous anticoagulation, and then were switched back to a non-vitamin $\mathrm{K}$ antagonist oral anticoagulant 1 to 2 months after ablation. This is because higher-risk patients, such as older patients with persistent atrial fibrillation, would have had a period of time before ablation and after sheath removal during which there was no anticoagulant present, which could increase the risk of an embolic event. However, the practice of switching anticoagulants is cumbersome for patients and physicians, and the growing use of non-vitamin $\mathrm{K}$ antagonist oral anticoagulants has made this approach impractical.

Cohort data suggest that interrupted and continuous non-vitamin $\mathrm{K}$ antagonist oral anticoagulants have similar safety and effectiveness in the context of ablation of atrial fibrillation. ${ }^{33}$ Interpretation of the available data is limited by small samples, small numbers of events, and nonrandomized or retrospective study designs. ${ }^{15-17}$ A recent meta-analysis including 7996 patients from 19 observational studies showed that nonvitamin $\mathrm{K}$ antagonist oral anticoagulants did not differ significantly from interrupted or continuous warfarin treatment with respect to the prevention of thromboembolic events but may be associated with a lower risk of overall bleeding and minor bleeding complications. ${ }^{18}$ The only prospective, randomized clinical trial performed to date was the VENTURE-AF trial. ${ }^{21}$ This controlled trial involving 248 patients undergoing ablation of atrial fibrillation showed that the uninterrupted use of a factor Xa inhibitor is feasible, with no meaningful difference in thromboembolic events or major bleeding events as compared with uninterrupted vitamin $\mathrm{K}$ antagonists. The major limitation of this trial was the small sample and low numbers of major bleeding events and thromboembolic events.

The major concern of performing catheter ablation with uninterrupted non-vitamin $\mathrm{K}$ antagonist oral anticoagulant therapy is the risk of bleeding, particularly life-threatening bleeding such as pericardial tamponade. In the present trial, the 
rate of major bleeding events was significantly lower in the dabigatran group than in the warfarin group. In particular, the rates of pericardial bleeding and groin hematoma were lower with dabigatran. The mechanism of reducing major bleeding events with dabigatran may be related to the more specific mechanism of action (direct thrombin inhibition rather than a decrease in the production of several coagulation factors) and shorter half-life of dabigatran as compared with warfarin, as well as the presence of normal levels of factor VII and a stable anticoagulation effect. Another potential advantage of the periprocedural use of dabigatran is the availability of idarucizumab, a dabigatran-specific reversal agent that can achieve an immediate and complete reversal of the anticoagulant effect. ${ }^{1}$ However, even though this agent became available during the RE-CIRCUIT trial, all the bleeding events in the dabigatran group were managed without the need for dabigatran reversal, which is reassuring. These outcomes are consistent with the results of the Randomized Evaluation of Long-Term Anticoagulant Therapy (RE-LY) trial, ${ }^{23}$ showing superiority of dabigatran (150 mg twice daily) versus a vitamin $\mathrm{K}$ antagonist, and of several robust and independent real-world studies that support the findings of the RE-LY trial. ${ }^{34,35}$

A potential limitation of our trial was the open-label design. The different dosing schedule of dabigatran, need for blood testing, and adjustments in the dose of warfarin made blinding clinically impractical. However, all outcome events were independently adjudicated by a blinded events committee. The sample size that would have been required for a formal noninferiority trial was prohibitive. However, the results observed, and the fact that dabigatran was ultimately superior to warfarin with respect to the primary end point, made our observations clinically relevant. We found that periprocedural anticoagulation with uninterrupted dabigatran (150 mg twice daily) was associated with fewer bleeding events than uninterrupted vitamin $\mathrm{K}$ antagonism.

\begin{abstract}
Supported by Boehringer Ingelheim.
Dr. Calkins reports receiving lecture fees and consulting fees from Medtronic and consulting fees from Abbott Laboratories and AtriCure; Dr. Willems, receiving honoraria from Bayer HealthCare, Bristol-Myers Squibb, Boston Scientific, Johnson \& Johnson, Medtronic, and Abbott Laboratories; Dr. Gerstenfeld, receiving grant support and honoraria from Biosense Webster and St. Jude Medical; Dr. Verma, receiving grant support and fees for serving on an advisory board from Bayer, grant support and lecture fees from Daiichi Sankyo, grant support from Biotronik, consulting fees from Biosense Webster, and research support and consulting fees from Medtronic; Dr. Schilling, receiving grant support, honoraria, and travel support from Biosense Webster, St. Jude Medical, Medtronic, and Boston Scientific; Dr. Hohnloser, receiving consulting fees, fees for serving on advisory boards, and lecture fees from Bayer HealthCare, Boston Scientific, Bristol-Myers Squibb, Cardiome Pharma, Forest Laboratories, Gilead Sciences, Johnson \& Johnson, Medtronic, Otsuka, Pfizer, Portola Pharmaceuticals, Sanofi-Aventis, Servier, St. Jude Medical, and ZOLL Medical; Dr. Okumura, receiving honoraria from Daiichi Sankyo, Bayer, Johnson \& Johnson, and Medtronic; Dr. Serota, receiving lecture fees from Novartis; Ms. Guiver, being a statistician for Boehringer Ingelheim; and Dr. Brouwer, receiving grant support from Bayer, Daiichi Sankyo, Bristol-Myers Squibb, and Pfizer. No other potential conflict of interest relevant to this article was reported.

Disclosure forms provided by the authors are available with the full text of this article at NEJM.org.

We thank Dr. Saulius Kalvaitis, who collaborated with Dr. Serota in the conduct of the trial; Dr. Victor Fernandez Gallego, Dr. Ernesto Ferreiros, Dr. Claudia Grauer, Dr. Ruth Harper, the clinical operations teams in the participating countries, and all clinical investigators for their valuable contributions; and Anoop
\end{abstract} Joseph, M.Sc., of Parexel for assistance with medical writing.

\section{REFERENCES}

1. Kirchhof P, Benussi S, Kotecha D, et al. 2016 ESC guidelines for the management of atrial fibrillation developed in collaboration with EACTS. Eur Heart J 2016;37:2893-962.

2. January CT, Wann LS, Alpert JS, et al. 2014 AHA/ACC/HRS guideline for the management of patients with atrial fibrillation: a report of the American College of Cardiology/American Heart Association Task Force on Practice Guidelines and the Heart Rhythm Society. J Am Coll Cardiol 2014;64(21):e1-e76.

3. Calkins H, Kuck KH, Cappato R, et al. 2012 HRS/EHRA/ECAS expert consensus statement on catheter and surgical ablation of atrial fibrillation: recommendations for patient selection, procedural techniques, patient management and follow- up, definitions, endpoints, and research trial design. Europace 2012;14:528-606.

4. Di Biase L, Burkhardt JD, Santangeli $\mathrm{P}$, et al. Periprocedural stroke and bleeding complications in patients undergoing catheter ablation of atrial fibrillation with different anticoagulation management: results from the Role of Coumadin in Preventing Thromboembolism in Atrial Fibrillation (AF) Patients Undergoing Catheter Ablation (COMPARE) randomized trial. Circulation 2014;129:2638-44.

5. Page SP, Siddiqui MS, Finlay M, et al. Catheter ablation for atrial fibrillation on uninterrupted warfarin: can it be done without echo guidance? J Cardiovasc Electrophysiol 2011;22:265-70.

6. Lakkireddy D, Reddy YM, Di Biase L, et al. Feasibility and safety of dabigatran versus warfarin for periprocedural anticoagulation in patients undergoing radiofrequency ablation for atrial fibrillation: results from a multicenter prospective registry. J Am Coll Cardiol 2012;59:1168-74.

7. Kim JS, She F, Jongnarangsin K, et al. Dabigatran vs warfarin for radiofrequency catheter ablation of atrial fibrillation. Heart Rhythm 2013;10:483-9.

8. Yamaji H, Murakami T, Hina K, et al. Usefulness of dabigatran etexilate as periprocedural anticoagulation therapy for atrial fibrillation ablation. Clin Drug Investig 2013;33:409-18.

9. Kaiser DW, Streur MM, Nagarakanti $\mathrm{R}$, Whalen SP, Ellis CR. Continuous warfarin versus periprocedural dabigatran to reduce stroke and systemic embolism in patients undergoing catheter ablation for 
atrial fibrillation or left atrial flutter. J Interv Card Electrophysiol 2013;37:241-7. 10. Bassiouny M, Saliba W, Rickard J, et al. Use of dabigatran for periprocedural anticoagulation in patients undergoing catheter ablation for atrial fibrillation. Circ Arrhythm Electrophysiol 2013;6: 460-6.

11. Nin T, Sairaku A, Yoshida Y, et al. A randomized controlled trial of dabigatran versus warfarin for periablation anticoagulation in patients undergoing ablation of atrial fibrillation. Pacing Clin Electrophysiol 2013;36:172-9.

12. Stepanyan G, Badhwar N, Lee RJ, et al. Safety of new oral anticoagulants for patients undergoing atrial fibrillation ablation. J Interv Card Electrophysiol 2014; 40:33-8.

13. Arshad A, Johnson CK, Mittal S, et al. Comparative safety of periablation anticoagulation strategies for atrial fibrillation: data from a large multicenter study. Pacing Clin Electrophysiol 2014;37:66573.

14. Armbruster HL, Lindsley JP, Moranville MP, et al. Safety of novel oral anticoagulants compared with uninterrupted warfarin for catheter ablation of atrial fibrillation. Ann Pharmacother 2015;49: 278-84.

15. Nagao T, Inden $Y$, Shimano $M$, et al. Feasibility and safety of uninterrupted dabigatran therapy in patients undergoing ablation for atrial fibrillation. Intern Med 2015;54:1167-73.

16. Lakkireddy D, Reddy YM, Di Biase L, et al. Feasibility and safety of uninterrupted rivaroxaban for periprocedural anticoagulation in patients undergoing radiofrequency ablation for atrial fibrillation: results from a multicenter prospective registry. J Am Coll Cardiol 2014;63:982-8. 17. Maddox W, Kay GN, Yamada T, et al. Dabigatran versus warfarin therapy for uninterrupted oral anticoagulation during atrial fibrillation ablation. J Cardiovasc Electrophysiol 2013;24:861-5.

18. Zhao Y, Yang Y, Tang X, Yu X, Zhang L, Xiao H. New oral anticoagulants compared to warfarin for perioperative anticoagulation in patients undergoing atrial fibrillation catheter ablation: a metaanalysis of continuous or interrupted new oral anticoagulants during ablation compared to interrupted or continuous warfarin. J Interv Card Electrophysiol 2017 January 12 (Epub ahead of print).

19. Wu S, Yang YM, Zhu J, et al. Metaanalysis of efficacy and safety of new oral anticoagulants compared with uninterrupted vitamin $\mathrm{K}$ antagonists in patients undergoing catheter ablation for atrial fibrillation. Am J Cardiol 2016;117:92634.

20. Heidbuchel H, Verhamme P, Alings $M$, et al. European Heart Rhythm Association practical guide on the use of new oral anticoagulants in patients with nonvalvular atrial fibrillation. Europace 2013; 15:625-51.

21. Cappato R, Marchlinski FE, Hohnloser $\mathrm{SH}$, et al. Uninterrupted rivaroxaban vs. uninterrupted vitamin $\mathrm{K}$ antagonists for catheter ablation in non-valvular atrial fibrillation. Eur Heart J 2015;36:180511.

22. Connolly SJ, Ezekowitz MD, Yusuf S, et al. Dabigatran versus warfarin in patients with atrial fibrillation. N Engl J Med 2009;361:1139-51.

23. Eikelboom JW, Wallentin L, Connolly SJ, et al. Risk of bleeding with 2 doses of dabigatran compared with warfarin in older and younger patients with atrial fibrillation: an analysis of the Randomized Evaluation of Long-Term Anticoagulant Therapy (RE-LY) trial. Circulation 2011; 123:2363-72.

24. Romanelli RJ, Nolting L, Dolginsky M, Kym E, Orrico KB. Dabigatran versus warfarin for atrial fibrillation in realworld clinical practice: a systematic review and meta-analysis. Circ Cardiovasc Qual Outcomes 2016;9:126-34.

25. Larsen TB, Rasmussen LH, Skjфth F, et al. Efficacy and safety of dabigatran etexilate and warfarin in "real-world" patients with atrial fibrillation: a prospective nationwide cohort study. J Am Coll Cardiol 2013;61:2264-73.

26. Graham DJ, Reichman ME, Wernecke $M$, et al. Stroke, bleeding, and mortality risks in elderly Medicare beneficiaries treated with dabigatran or rivaroxaban for nonvalvular atrial fibrillation. JAMA Intern Med 2016;176:1662-71.

27. Graham DJ, Reichman ME, Wernecke
$\mathrm{M}$, et al. Cardiovascular, bleeding, and mortality risks in elderly Medicare patients treated with dabigatran or warfarin for nonvalvular atrial fibrillation. Circulation 2015;131:157-64.

28. World Medical Association. World Medical Association Declaration of Helsinki: ethical principles for medical research involving human subjects. JAMA 2013;310:2191-4.

29. ICH harmonised tripartite guideline. Guideline for Good Clinical Practice E6(R1) (http://www.ich.org/fileadmin/Public_ Web_Site/ICH_Products/Guidelines/ Efficacy/E6/E6_R1_Guideline.pdf).

30. Rosendaal FR, Cannegieter SC, van der Meer FJ, Briët E. A method to determine the optimal intensity of oral anticoagulant therapy. Thromb Haemost 1993; 69:236-9.

31. Schulman S, Kearon C, Subcommittee on Control of Anticoagulation of the Scientific and Standardization Committee of the International Society on Thrombosis and Haemostasis. Definition of major bleeding in clinical investigations of antihemostatic medicinal products in nonsurgical patients. J Thromb Haemost 2005; 3:692-4.

32. Sardar P, Nairooz R, Chatterjee S, Wetterslev J, Ghosh J, Aronow WS. Metaanalysis of risk of stroke or transient ischemic attack with dabigatran for atrial fibrillation ablation. Am J Cardiol 2014;113: 1173-7.

33. Aryal MR, Ukaigwe A, Pandit A, et al. Meta-analysis of efficacy and safety of rivaroxaban compared with warfarin or dabigatran in patients undergoing catheter ablation for atrial fibrillation. Am J Cardiol 2014;114:577-82.

34. Larsen TB, Skjфth F, Nielsen PB, Kjældgaard JN, Lip GY. Comparative effectiveness and safety of non-vitamin $\mathrm{K}$ antagonist oral anticoagulants and warfarin in patients with atrial fibrillation: propensity weighted nationwide cohort study. BMJ 2016;353:i3189.

35. Yao X, Abraham NS, Sangaralingham LR, et al. Effectiveness and safety of dabigatran, rivaroxaban, and apixaban versus warfarin in nonvalvular atrial fibrillation. J Am Heart Assoc 2016;5(6):e003725.

Coppright $\odot 2017$ Massachusetts Medical Society. 